\title{
El desarrollo local y el bienestar de la ciudadanía: ¿una relación necesaria?
}

\author{
Teresa Torns, Laia Castelló, Carolina Recio Cáceres \\ Universitat Autònoma de Barcelona
}

Centre d'Estudis Sociològics sobre la Vida Quotidiana i el Treball (QUIT)

teresa.torns@uab.cat; laia.castello@uab.cat; carolina.recio@uab.cat

\section{Resumen}

El artículo presenta unas reflexiones sobre el vinculo que existe entre las politicas de bienestar y el desarrollo local en España. Para ello trata de: a) discernir hasta qué punto la idea de bienestar forma parte del imaginario y de las prácticas de los ayuntamientos; b) delimitar el espacio que esa idea y esas prácticas ocupan en la agenda municipal; $y$ c) apuntar algunas de las razones por las cuales la relación entre bienestar, politica local y empleo de calidad parecen ser casi inexistentes.

Palabras clave: desarrollo local, bienestar cotidiano, trabajo de cuidado, empleo, SAD.

\begin{abstract}
The article presents some reflections on the link between social care and local development in Spain. It attempts to: a) determine the extent to which the idea of wellbeing is part of the social imaginary and the practices of local councils; $b$ ) define the importance that this idea and these practices bave in the local agenda; and c) reveal some of the reasons why the relationship between care, local politics and quality employment seems to be almost nonexistent.
\end{abstract}

Keywords: local development, daily wellbeing, care work, employment, home care services. 


\section{Introducción}

El artículo refleja parte de los hallazgos de la investigación «Desarrollo Local, Social y Sostenible, con Generación de Empleo-DELOGE ${ }^{1} \gg$. En concreto, resume los resultados relacionados con una de las hipótesis principales del proyecto, orientada a comprobar si existía un modelo alternativo de desarrollo local. Según esa hipótesis, este modelo tenía que ser capaz de incorporar aspectos relacionados con el bienestar de la ciudadanía y la sostenibilidad ambiental en los planes estratégicos municipales, y de contribuir, además, a la generación de empleo de calidad en el sector de servicios de atención a las personas en el ámbito local.

A tenor de los resultados obtenidos, el artículo presenta unas primeras conclusiones sobre la relación actual entre las políticas locales de bienestar y los planes de desarrollo local. Para llevar a cabo ese cometido, en primer lugar se resumen los datos generales que han enmarcado la investigación, así como el contexto geográfico y socioeconómico de los municipios analizados. En segundo lugar, se plantean algunos de los argumentos que sustentan teóricamente el análisis de los resultados. En especial, aquellos que remiten a la relación entre los objetivos convencionales del desarrollo local y el ámbito del bienestar cotidiano de la ciudadanía.

En tercer lugar, se detallarán los principales resultados obtenidos del análisis del discurso de los agentes locales sobre el mencionado bienestar, concretados en las políticas de atención a las personas. Este discurso, pese a haber ganado protagonismo en esta última década, no parece haber encontrado su correlato en la agenda de las políticas municipales. Los datos observados apuntan hacia unas actuaciones en el ámbito de los servicios personales fuertemente deudoras de la tradición asistencialista o, cuando menos, lastradas por el estigma de la beneficencia. Así mismo, el análisis del imaginario social, proyectado en esos discursos, pone de manifiesto la persistencia de la división sexual del trabajo; una persistencia que ignora el aporte al bienestar cotidiano que supone el trabajo invisible de las mujeres en las familias, una situación que se ve agravada en una sociedad como la española, donde la mezcla de los regímenes de bienestar y de género se asienta en una fuerte tradición familista, en la cual los servicios de atención a la vida diaria son, todavía, rara avis.

Como consecuencia, la promoción de este tipo de políticas sociales no forma parte de los planes de promoción económica de los municipios. Y como a pesar de que tales servicios de bienestar son una de las principales vías de generación de empleo a escala local, paradójicamente, la promoción del desarrollo local no las contempla, o las considera como políticas de cohesión social destinadas tan sólo a grupos en riesgo de

1 Esta investigación fue realizada por los miembros del Centre d'Estudis Sociològics sobre la Vida Quotidiana i el Treball del Departamento de Sociología de la UAB (QUIT), bajo la dirección de Fausto Miguélez. La investigación ha sido financiada por el Ministerio de Educación y Ciencia (Plan Nacional I+D+I - SEJ2005-04078). 
exclusión social, principalmente mujeres adultas con dificultades para encontrar un empleo, dado su bajo nivel de formación o su pertenencia a la población inmigrada. Éstas son unas conductas que olvidan las posibilidades de un yacimiento de empleo, detectado ya hace casi dos décadas, e impide la profesionalización y revalorización de un trabajo absolutamente indispensable para el cuidado de la vida y el bienestar de la ciudadanía.

En resumen, el texto pone de manifiesto la coexistencia de dos procesos que parecen ser contradictorios, en la relación entre el desarrollo local y el ámbito de las políticas de bienestar. Por un lado, el impulso relativo de las políticas de bienestar plasmado en la generalización de un discurso universalista, tal como puede deducirse del reciente marco legislativo. Y por otro, las prácticas reales, que señalan cómo el bienestar de la ciudadanía se sigue contemplando como un aspecto marginal de las agendas políticas municipales. Esta contradicción se traduce en la persistencia de un modelo asistencialista de bienestar, para el cual las políticas de desarrollo local no tienen en cuenta ni los valores relacionados con el bienestar cotidiano ni, por descontado, la posibilidad de generar un sector con empleo de calidad.

\section{El contexto general de la investigación}

La investigación DELOGE se realizó en dos etapas. La primera tuvo lugar a lo largo del año 2006 y la segunda, entre los años 2007 y 2008. Durante la primera etapa se realizaron entrevistas (16 en total) a técnicos municipales de las diferentes áreas de gobierno de los municipios seleccionados. El objetivo de aquella etapa fue la realización de informes acerca de la situación socioeconómica de los territorios escogidos: Sant Feliu de Llobregat, Molins de Rei y Manresa. Estos informes facilitaron el diseño del trabajo de campo de la segunda etapa. Ésta consistió en la realización de 39 entrevistas en profundidad a personas de los tres campos de interés de la investigación DELOGE: la promoción económica, el medio ambiente y el sector de atención a las personas. Las 55 personas entrevistadas, en el total de las dos etapas, eran «expertas» y/o informantes clave de los tres municipios. Finalmente, se realizaron entrevistas informativas a los agentes locales: representantes de administraciones supralocales, de organizaciones empresariales y sindicales y de colegios profesionales.

En relación con la temática aquí referenciada, debe precisarse que en el momento en que se realizó la segunda fase de la investigación se estaba poniendo en marcha la Ley de Promoción de la Autonomía Personal y Atención a las Personas en Situación de Dependencia y la Ley de Servicios Sociales de Cataluña. Esta cuestión es crucial para entender los resultados obtenidos en las entrevistas, ya que este nuevo marco le- 
gal introdujo modificaciones en el panorama y organización de los servicios sociales y personales en los municipios. En concreto, en todo lo relacionado con los Servicios de Atención a Domicilio (SAD), unos servicios de proximidad que, tal como pensamos, debían configurar un sector con potencialidad para generar empleo y para revisar los planteamientos del bienestar de la ciudadanía. La investigación DELOGE coincidió, así, con un momento histórico que por fuerza ha marcado los discursos obtenidos, tal como se verá en el análisis de los resultados.

\subsection{Las características del territorio}

Sant Feliu de Llobregat y Molins de Rei son municipios colindantes y están situados en el cinturón industrial de Barcelona. Por el contrario, Manresa está en la Cataluña central, alejada del área de influencia de la capital. El tamaño de los mismos es dispar. Manresa tiene en torno a 72.000 habitantes, Sant Feliu de Llobregat cuenta con 42.000 y Molins de Rei traspasa levemente los 23.000 habitantes $^{2}$. Sin embargo, las tres poblaciones presentan dinámicas parecidas: una gran tradición en la recepción de población inmigrante y un notorio proceso de envejecimiento de la población. De hecho, los tres municipios están viendo aumentada su población desde el año $2000^{3}$ debido a la llegada de inmigrantes extranjeros y de personas jóvenes procedentes de la capital catalana, atraídas por un precio más asequible de la vivienda. Así mismo, cabe destacar el proceso de envejecimiento porque, aunque no es un proceso exclusivo de estos tres municipios, les está obligando a desarrollar soluciones públicas para dar atención a este colectivo, con políticas pensadas desde la proximidad. Finalmente, los tres municipios comparten un fuerte legado industrial, que se ha plasmado en una tasa de actividad mayor que la media catalana; actividad laboral en la que se observa, también, una alta tasa de actividad femenina que, en la actualidad, se concentra en el sector servicios, dada la transformación de la estructura productiva ocurrida en estas últimas décadas.

2 Estos datos provienen de la muestra continua de los padrones municipales que proporciona el Institut Català d'Estadística (IDESCAT).

3 La población de Manresa ha aumentado en diez años (1998-2008) el 17\%; un 25\% en Molins de Rei, y otro 17\% en Sant Feliu de Llobregat. 


\section{El desarrollo local y el bienestar de la ciudadanía: ¿una relación necesaria?}

Frente a aquellos a quienes pueda parecer ilícita o poco pertinente la necesidad de establecer una relación como la que encabeza este apartado, podemos aportar diversas razones que avalan esa necesidad, la mayoría de ellas procedentes de los estudios que demuestran la necesidad de aportar bienestar cotidiano a la ciudadanía. Este fenómeno va en aumento, dados los cambios surgidos en las sociedades en que el modelo social europeo cobra sentido, lugares donde las políticas sociales del estado del bienestar contemplan esa necesidad como un derecho de ciudadanía, independientemente de la situación laboral y familiar de las personas.

Esa creciente necesidad de aporte de bienestar cotidiano proviene principalmente, según los especialistas, del envejecimiento de la población, de los cambios en la estructura familiar patriarcal y de la mayor participación de las mujeres en el mercado laboral. Se trata de unos cambios de carácter local y global, mucho más agudizados en las sociedades mediterráneas, donde la debilidad del estado del bienestar es mayor y la tradición familista fuerte, y en donde tales cambios requieren la búsqueda urgente de nuevas formas de organizar socialmente el trabajo de cuidado cotidiano (Torns, 2008) entre las instituciones básicas de transferencia de bienestar: el mercado, el Estado y la familia, ya que, ante la desestabilización de las formas tradicionales de provisión del bienestar cotidiano, no cuentan ni con los medios materiales suficientes ni con los imaginarios sociales adecuados para afrontar esa situación.

Por encima de otras consideraciones, parece fuera de duda que los factores sociodemográficos se encuentran entre los principales inductores de esos cambios. Hace ya casi veinte años, los especialistas europeos que encontraron los llamados «yacimientos de empleo» alertaron sobre la falta de mujeres de generaciones contiguas (45-65 años) necesarias para afrontar el cuidado; un agujero demográfico producido por el aumento del envejecimiento de la población y la baja natalidad, fenómeno especialmente alarmante en los países mediterráneos, que ha sido posteriormente calificado desde el mundo anglosajón como caring gap (Anderson, 2000). Ese fenómeno acontece, además, en un escenario donde, desde voces favorables a las políticas de igualdad entre géneros, se cuestiona la unilateralidad femenina en el suministro cotidiano de ese bienestar, una unilateralidad no debida al hecho de que las mujeres se hayan incorporado, más o menos recientemente, al mercado de trabajo, tal como dicen quienes ignoran la tradición femenina de trabajo industrial o su apabullante presencia en el servicio doméstico, sino originada por la crisis del modelo «hombre ganador de pan/mujer ama de casa». Este modelo ha sido el sustentador invisible del bienestar cotidiano en el actual estado del bienestar y, en la actualidad, a pesar de haber sido considerado como 
un modelo en declive (Lewis, 2001), está lejos de encontrar su alternativa. En primer lugar, por la falta o insuficiente colaboración de los hombres en el ámbito doméstico (González, 2000) y, muy especialmente, por la insuficiente organización social del trabajo de cuidado cotidiano por parte del Estado, en países como el nuestro. En este sentido, cabe reseñar el avance que supone la promulgación y aplicación de la nueva legislación española y catalana antes mencionada, en particular la mal denominada Ley de Dependencia, como acicate para mejorar algunos aspectos relacionados con la provisión del bienestar cotidiano; aunque el despliegue inicial de esas leyes no parece aportar demasiado optimismo a la cuestión.

De hecho, puede afirmarse que, para lograr que ese nuevo horizonte en que la organización social del cuidado cotidiano sea una realidad, se requiere, entre otras cuestiones, erosionar unos esquemas de pensamiento profundamente arraigados en la tradición productivista de los derechos de ciudadanía, unos derechos en los que la contribución laboral del cabeza de familia es la condición obligatoria para que él y su familia reciban protección social. Así mismo, debe considerarse que alcanzar una organización social del bienestar cotidiano satisfactoria supone desarrollar plenamente unos servicios de proximidad, de atención a la vida diaria o servicios de atención a domicilio (SAD), nombres diversos con los que en España bautizamos a los care services. Se trata de unos servicios que, lejos de contar con una demanda social ampliamente explicitada, deben afrontar, también, las resistencias culturales que subyacen en el imaginario social que preside el trabajo de cuidado: carencias y estereotipos que provocan que tales servicios no sean vistos como necesarios, dado que las tareas de cuidado todavía son contempladas como algo propio de la familia y, en consecuencia, como algo que se mueve de manera ambigua entre la actividad y las emociones que las mujeres deben asumir $y$, de hecho, asumen como obligación moral.

Por otra parte, nadie niega la incidencia que tienen en la crisis o declive del modelo de contrato social entre hombres y mujeres en las sociedades del bienestar (Gardiner, 2000; Pateman, 1994) el aumento de la presencia de las mujeres de clase media en el mercado de trabajo, así como las transformaciones sufridas por la familia patriarcal; $y$, en consecuencia, la influencia que todo ello tiene en la provisión de bienestar cotidiano de la población. No obstante, debe precisarse que esa mayor presencia femenina en el mercado de trabajo ha provocado un aumento de las desigualdades de género en el propio mercado: ha concentrado a las mujeres en empleos no cualificados, mayoritariamente en los servicios a las personas, que son necesarios para proveer bienestar cotidiano, pero se han configurado como guetos mal remunerados y desprestigiados, lo que ha conducido a la mayoría de mujeres ocupadas, incluso a las más privilegiadas, a vivir en régimen de «doble presencia» (Balbo, 1994), complicándoles la gestión y ejecución del trabajo doméstico y de cuidado que deben asumir para atender sus supues- 
tas obligaciones familiares. De hecho, esas dificultades femeninas explican, de manera un tanto simplista pero más nítidamente que otros factores, el aumento de la demanda de trabajo de cuidado en el seno del hogar-familia, un incremento que suele resolverse, en España, a través de la contratación de mujeres inmigradas (Bettio y Plantenga, 2004; Bettio et alii, 2006) en la mayoría de las ocasiones, lo que acaba engrandeciendo la economía sumergida, o a través, en el resto, de los escasos recursos que ofrecen las instituciones públicas (servicios SAD) o entidades privadas del tercer sector.

\subsection{El trabajo de cuidado y la provisión de bienestar cotidiano}

En el campo de la sociología y de forma generalizada, a menudo se emplean, en época relativamente reciente, los términos «cuidado» $y$ «trabajo de cuidado» de manera indistinta y un tanto arbitraria (Ungerson, 2005). Como se ha apuntado en otros escritos, (Torns, 2008), al aplicar el término «cuidado» de manera aislada se corre el riesgo de olvidar los puntos de conexión de esta actividad con el mundo del trabajo. Lo desvincula de la ruptura conceptual de los años ochenta, que ensanchó el concepto de trabajo más allá de las relaciones salariales y permitió aflorar el trabajo doméstico y familiar; y, lo más importante, tiende a esencializar la relación entre el cuidado (del que supuestamente sólo son capaces las mujeres) y el bienestar cotidiano. Los motivos que explican esta situación parecen radicar en el hecho de que el concepto actual de cuidado nace de los análisis de los límites del estado del bienestar desde una perspectiva de género, y no desde la sociología del trabajo, donde existe escasa tradición sociológica en estudiar las implicaciones que el trabajo tiene en relación al bienestar.

Así las cosas, parece oportuno recordar que la aportación básica del trabajo doméstico y familiar, del que formaría parte el «trabajo de cuidado», es la provisión de bienestar cotidiano a las personas que conviven en el núcleo familiar, franqueando el umbral del escenario físico y simbólico del hogar, así como facilitar la disponibilidad laboral de los adultos masculinos del hogar (Torns, 2008). Estas finalidades avalan, a nuestro parecer suficientemente, la afirmación central que justifica la hipótesis de nuestra investigación: el trabajo de cuidado resulta indispensable para el bienestar cotidiano de las personas en particular, y de la sociedad en general, por lo que debe colegirse, en consecuencia, que ese bienestar debe ser organizado socialmente, a partir de los cambios experimentados en las sociedades del bienestar, tal como hemos referenciado en párrafos anteriores. $\mathrm{Y}$ esa organización social del cuidado cotidiano debe suponer el impulso y la promoción de los servicios capaces de procurar el bienestar cotidiano de la población, sea cual sea su situación familiar y laboral. Ello significa promover y amparar la creación de servicios SAD de calidad, y además, considerar el ámbito local 
como escenario de proximidad idóneo para el desarrollo de unos derechos básicos de ciudadanía, en clave universal e individualizada. Por lo tanto, el desarrollo local debe contemplar los servicios SAD como uno de sus ejes prioritarios, tanto por el aumento de empleo que suponen como por su enorme implicación en la promoción del bienestar de la ciudadanía.

\subsection{El desarrollo local como escenario del bienestar}

Tal como se ha comentado en la introducción, las hipótesis de nuestra investigación suponían la existencia de un nuevo modelo de desarrollo local (DL), un modelo cuya delimitación requería ampliar la noción clásica de desarrollo, basada, generalmente, en el crecimiento económico y la lógica industrial de los territorios.

El modelo alternativo incorporaría, así, los nuevos discursos sobre el desarrollo local que recogen las reflexiones sobre el bienestar cotidiano y la posibilidad de introducirlas en las políticas municipales. Esta nueva propuesta de desarrollo local también se apoya en la premisa de lograr crecimiento económico, pero introduce límites basados en la sostenibilidad ecológica (Renner, 2000; González, 2000; Allende, 1995) y la equidad social. Es decir, lejos de preconizar el crecimiento económico per se, se contempla un desarrollo local que tiene en cuenta las nuevas necesidades sociales y ecológicas de los territorios. Ello obliga, además, a buscar nuevas formas de diseñar y promover políticas, capaces de impulsar un cambio progresivo de los imaginarios sociales relacionados con el desarrollo, el bienestar y la proximidad. Este enfoque surge, en definitiva, del deseo de superar algunas deficiencias del modelo de desarrollo clásico (basado en el modelo taylorista-fordista), que no podía hacer frente a la nueva situación socioeconómica de los municipios.

Otro de los elementos de cambio en el nuevo DL es el papel de la Administración local, ya que en el modelo clásico los procesos de desarrollo estaban liderados por la Administración central, mientras que los gobiernos locales quedaban en segundo término. En este contexto nuevo, la Administración local será la encargada de liderar los procesos de desarrollo local. También los actores sociales de los territorios adquieren un papel más destacado, puesto que se ponen en marcha procesos de participación entre las autoridades locales, las empresas y la comunidad con el objetivo de lograr nuevas áreas de crecimiento en los municipios. Todo ello conduce finalmente a que este nuevo DL contemple la utilización de los recursos endógenos del municipio como característica principal (Vázquez, 1999), lo que significa poner especial atención en las nuevas necesidades sociales y medioambientales, impulsar una cultura local innovadora y promover procesos de participación ciudadana que actúen como guardianes y garantes 
del proceso de cambio, con el añadido, no banal, de tener como objetivo primordial la generación de nuevos espacios para el desarrollo de sectores productivos, capaces de generar empleo de calidad.

\section{El análisis de los resultados}

Los resultados de la investigación, aquí reseñados, remiten exclusivamente al análisis de los discursos de los agentes locales sociales que tratan de dar respuesta a las hipótesis iniciales. En concreto, tratamos de discernir qué tipo de discurso preside las políticas y actuaciones locales relacionadas con el bienestar cotidiano de la ciudadanía, $y$ de averiguar si tales actuaciones han producido un aumento significativo del empleo y, más específicamente, del empleo de calidad en el sector de atención a las personas (SAD).

\subsection{Servicios sociales y políticas municipales: un largo recorrido desde el asistencialismo al universalismo}

Uno de los datos más destacables entre los resultados obtenidos es que los servicios sociales y personales tienden a gozar de más y mejor consideración en el conjunto de las políticas municipales. Incluso puede afirmarse que, hoy en día, los programas, todavía llamados de asistencia social, suelen pensarse ya como políticas universales. Ahora bien, pese a estos esfuerzos, son numerosos los factores que muestran la persistencia de la lógica asistencialista en este tipo de políticas.

Sin embargo, la importancia cada vez mayor de las políticas de atención a las personas en el conjunto de las políticas municipales tiene efectos cada vez más visibles sobre el volumen y calidad de los servicios y, cómo no, sobre el empleo presente y futuro de los municipios. Así, en esta última década se han reforzado este tipo de servicios debido, primordialmente, al aumento de población envejecida. En concreto, se trata de servicios que se prestan en el hogar de las personas usuarias porque, desde el discurso político, se asegura la idoneidad de no separar a las personas de su contexto cotidiano, un hecho que ha provocado que, en todos los territorios estudiados, los SAD hayan ido incrementando tanto el número de servicios prestados como el número de personas ocupadas en este sector. Este aumento va ligado, de manera clara, a la interiorización creciente del discurso universalista entre los responsables de la política municipal, un discurso que defiende el derecho de ciudadanía a la hora de recibir los servicios destinados a cubrir las necesidades de cuidado. Ello explicaría, en buena medida, el cambio 
del papel e importancia de las áreas municipales de servicios sociales y personales a lo largo de los últimos años, un cambio que todas las personas entrevistadas en los municipios perciben como cierto, puesto que hasta hace pocos años estos servicios permanecían casi olvidados en la agenda municipal. Según lo relatado, la función de aquellos servicios era atender tan sólo los problemas de un núcleo de población excluida, con unas demandas y necesidades sociales apenas visibles. El cambio de discurso, sin duda, ha sido también propiciado por el desarrollo del reciente marco legislativo español y catalán, una legislación que ha obligado a acelerar el despliegue de estos servicios en clave de derecho universal e individual. Aun así, tal y como nuestra investigación ha podido comprobar, este despliegue se está llevando a cabo con ritmos diferentes y con modelos distintos, lo que supone que cada municipio ha ido dibujando de forma distinta, en función de sus capacidades financieras y voluntades políticas, sus servicios (SAD), sin un modelo de referencia común. En estos momentos, sin embargo, parece que el modelo de servicios sociales y personales está consolidándose, y todo parece apuntar hacia la convergencia de un modelo común, aquél que considera el SAD y la tele-asistencia como servicios prioritarios, de carácter universal, tal y como recoge la ley; en definitiva, a la comprensión de que es obligatorio atender las situaciones de gran dependencia a través de los servicios públicos.

Así pues, en los tres municipios analizados se percibe un cambio de rumbo hacia la mejora del reconocimiento y el prestigio del área de servicios sociales y personales dentro de la Administración local, al menos a escala del discurso político y técnico. No obstante, las prácticas o, si se quiere, las formas en que se concretan las políticas, parecen seguir apuntando hacia una persistencia del asistencialismo, y hacia la invisibilidad de las actuaciones que ponen el bienestar cotidiano en el centro de esas políticas. A ello debe añadirse que estas políticas están afectadas por graves problemas de financiación: la dificultad para poner en marcha y ampliar servicios sin el apoyo financiero adecuado proveniente de las administraciones supramunicipales, es una de las quejas persistentes del personal técnico de los diversos ayuntamientos. En este sentido, todas las personas entrevistadas coinciden en denunciar la demora en la recepción de las ayudas, y no parece descabellado pensar que el momento actual de crisis económica se perfila como un freno para el desarrollo definitivo de estas áreas.

De hecho, los datos correspondientes a las tasas de cobertura de estos servicios muestran que continúan siendo muy bajas. Según los datos que ofrece el Mapa de Servicios Sociales de Cataluña, en 2007 la media de horas de atención domiciliaria, entre la población $\geq 65$ años, era sólo de 3,19 horas semanales. Parece, pues, evidente que la lógica universalista se ha asumido desde el discurso, pero no se corresponde con la práctica, y que, pese al cambio impulsado por la legislación y los discursos de los agentes locales, la tradición familista y el asistencialismo de los servicios sociales conti- 
núan presidiendo la realidad de la atención y cuidado de la vida de las personas tanto en Cataluña como en el resto de España; o, lo que viene a ser lo mismo, la provisión del bienestar cotidiano de la población continua apoyándose en las familias más que en los servicios públicos.

\subsection{El empleo en los SAD y las políticas de desarrollo local}

Las hipótesis de partida también planteaban comprobar qué tipo de empleo se había generado en este sector, cómo lo había hecho y hasta qué punto esas políticas formaban parte de los planes de promoción económica. De nuevo debe recordarse que son los discursos de los agentes sociales los que guían los resultados aquí reseñados. Según esos discursos, se constata que el sector de servicios a las personas está creciendo, lo que conlleva creación de nuevo empleo en ese sector. Pero tal constatación no significa que se haya pensado en ese sector como un elemento crucial en los planes de desarrollo local.

Existen una serie de factores que resultan determinantes para la promoción o no de empleo de calidad en el sector de los SAD. En primer lugar, la importancia que el equipo de gobierno municipal confiere al sector $y$, principalmente, la relación que mantiene el Área de servicios sociales y personales con otras áreas de gobierno. La existencia de un vínculo entre el área de esos servicios y las áreas de promoción económica y de empleo parece ser la clave en ese proceso ya que, como parece obvio, sólo así se pueden incorporar los SAD a los planes de desarrollo local, dada la tradición economicista que preside ese tipo de desarrollo. No obstante, conviene no olvidar que este nuevo empleo, generado en numerosos lugares sólo gracias al desarrollo de los SAD, tropieza a menudo con lógicas que escapan a la dinámica del DL de los propios municipios, pues sucede que, aunque los gobiernos locales son los detectores del aumento de las necesidades sociales, no pueden intervenir ni en la definición del perfil de los profesionales requeridos, ni en la asunción de los costes que ello implica. Esto provoca que los perfiles dependan de los contenidos y la valoración de unos puestos de trabajo de baja cualificación, que son las características primordiales de este tipo de servicios; y que, además, esos mismos servicios $\mathrm{SAD}$ se vean sometidos a unos procesos de privatización que los derivan hacia las empresas privadas que operan en este sector. Todos estos factores, si bien permiten observar la existencia de cierta relación entre la generación de empleo y el despliegue de los SAD en los municipios, difícilmente permiten establecer unos vínculos entre este empleo y las actuaciones específicas del DL; y, mucho menos, decir que se trata de empleo de calidad, una situación que refuerza, una vez 
más, la lógica asistencialista y familista que preside la provisión de bienestar cotidiano en nuestro país.

\subsection{Las empresas de los servicios sociales de atención a la vida diaria $(\mathrm{SAD})$}

El actual modelo de prestación de los SAD en Cataluña y España es de titularidad pública, pero se desarrolla mayoritariamente a través de operadores externos, ya sean empresas privadas o del tercer sector. Es decir, hay provisión pública y gestión privada. Esta situación, en principio, da sentido a la creciente proliferación de alternativas locales de fomento y expansión del tejido empresarial en estos sectores, y configura los SAD como un nicho de mercado importante. En otras palabras, el sector de los servicios de atención a las personas es un sector económico en expansión que, en principio, ofrece cierta tendencia hacia la estabilidad y la seguridad en el empleo, dada su alta demanda y su relación con el sector público, pero que, paradójicamente, no logra que los empleos generados en los SAD salgan del gueto de la baja cualificación y salarios.

La manera de desarrollar los $\mathrm{SAD}$, según hemos podido analizar en cada uno de los ayuntamientos estudiados, es la concesión administrativa del servicio a una o más empresas que se presentan como candidatas en un concurso público. Los ayuntamientos publican un pliego de condiciones o conjunto de documentos que recogen las características mínimas que deben respetar las empresas que quieran prestar el servicio municipal. Ese pliego suele fijar el precio-hora del servicio, el número de profesionales necesarios y la obligatoriedad de respetar el convenio colectivo del sector, entre otros aspectos; es el modelo que se sigue en los tres casos analizados. Es un sistema pensado para conseguir el abaratamiento de los costes del servicio, pero también para simplificar el sistema de control y organización de un servicio de las características del SAD.

Este sistema abre la puerta al desarrollo de la actividad económica en este sector, que en principio parecería orientado a la creación de empresas de ámbito local, por lo que podría ser visto como una forma de promoción de desarrollo local, tal y como ha sido entendido en el conjunto de la investigación aquí presentada. Aun así, los resultados obtenidos parecen ir en un sentido contrario, ya que, de igual manera a lo que está sucediendo en el conjunto de España, son las medianas y grandes empresas las que están absorbiendo el volumen y la gestión de este tipo de servicios. En concreto, en 2006, la cobertura de este tipo de servicios en España estaba concentrada en sólo trece compañías, que tenían la Administración Pública como principal cliente. Y aunque es un sector con un gran número de operadores, en general destacan las organizaciones del tercer sector. Debe añadirse, además, que el futuro parece apuntar, cada vez más, hacia 
la concentración en grandes empresas, ya que ellas serán las únicas capaces de ajustarse mejor a los precios que fija la Administración local, así como a hacer frente a las infraestructuras necesarias para abarcar todo el volumen de servicios que se prevé y a la complejidad de gestión que este volumen requiere (Miguélez et alii, 2006; Miguélez y Lope, 2007). Debe añadirse, así mismo, que así como en otras áreas productivas se han hecho esfuerzos para la creación de viveros de empresas, o clústeres, las iniciativas en materia de servicios de atención a las personas son más limitadas. En concreto, parece quedar claro que, en relación con los $\mathrm{SAD}$, los municipios contratan a aquellas empresas que mejor se adaptan al precio público fijado por el Ayuntamiento y que ofrecen más garantías para cubrir los servicios, sin preocuparse demasiado por la calidad del empleo creado ni del servicio ofrecido, circunstancias que favorecen el que los SAD sólo puedan ser asumidos por las empresas de servicios con un número de trabajadores elevados. Son, éstas, empresas que se caracterizan, además, por una gestión extremadamente flexible de sus recursos humanos y materiales, los bajos salarios que ofrecen y una alta rotación laboral de una mano de obra mayoritariamente femenina.

\section{4 ¿Hacia un empleo de calidad?}

Los resultados reseñados hasta el momento permiten constatar que los SAD son un sector en clara expansión que, además, está generando empleo y ofreciendo oportunidades para la creación de empresas en los distintos territorios. Veamos ahora qué tipo de empleo se ha creado y si se trata o no de empleo de calidad. La carencia de profesionales del sector ha sido una queja recurrente en todas las entrevistas realizadas, y ha sido expresada por técnicos, políticos y profesionales del sector. En este punto, parece oportuno, pues, analizar cuál es la estrategia escogida por los municipios a la hora de impulsar el empleo ligado a los SAD.

El análisis de las entrevistas nos permite afirmar que los tres municipios estudiados han establecido pequeñas colaboraciones con las diferentes áreas del gobierno municipal, especialmente con las de empleo. La necesidad urgente de profesionales, en concreto de trabajadoras familiares y auxiliares de geriatría, ha sido la piedra de toque de esta colaboración, y la actuación conjunta más habitual ha consistido en elaborar planes de formación profesional destinados a cubrir estos perfiles ${ }^{4}$. Según las entrevistas realizadas, parece claro que, en la actualidad, todavía continúa vigente aquel modelo. Es decir, las actuaciones municipales consisten, por un lado, en la tramitación de los expedientes para recibir las prestaciones asignadas (al Departamento de Servicios So-

4 Cabe decir que estas actuaciones todavía son deudoras de las iniciativas sujetas a los denominados yacimientos de empleo, unas iniciativas que, desde la década de 1990, han formado parte de las políticas de empleo locales con la finalidad de hacer frente a las altas tasas de paro, particularmente femeninas. 
ciales), y, por el otro, en la realización de cursos de formación ocupacional para formar profesionales del sector (en el Departamento de Empleo o Promoción Económica). La relación que se establece entre ambos departamentos es que el primero proporciona las personas que seguirán los cursos de formación ocupacional, que serán organizados por el segundo.

Pero debe destacarse que, muy frecuentemente, esta colaboración se entiende más como una medida dirigida a fomentar la cohesión o inclusión social que como una medida orientada a la promoción del desarrollo local. En particular, porque se trata del sector de los servicios SAD y las protagonistas de los cursos de formación son, según la lógica laboral que preside esos servicios, mujeres poco cualificadas o de difícil inserción laboral. La constatación de estos resultados ha hecho tambalear la línea argumental que sustentaba nuestras hipótesis de partida. $\mathrm{O}$, lo que parece igual pero no es lo mismo, su comprobación se verifica en sentido a contrario a la propuesta planteada.

En este punto, conviene precisar que el modelo empleado en el sector SAD, en los municipios analizados, responde a una estrategia específica que impide la relación positiva entre los SAD y la creación de empleo de calidad. En consecuencia, dificulta que los SAD sean contemplados como eje prioritario del desarrollo local. Corrobora tales afirmaciones el hecho innegable de que han proliferado planes de empleo para dar respuesta a colectivos concretos del mercado laboral, grupos a los que se considera con dificultades para insertarse en el mercado de trabajo. Se trata, como ya se ha comentado, de personas con baja cualificación, especialmente mujeres de mediana edad. Muchas de ellas se encuentran en situación de exclusión social, con una necesidad urgente de encontrar un trabajo remunerado y sometidas, en su entorno familiar, a la división sexual del trabajo tradicional. Por otro lado, los imaginarios sociales vigentes continúan vinculando este tipo de servicios SAD con el trabajo doméstico y familiar que realizan las mujeres y el servilismo (Fraisse, 2001), un trabajo que, debemos recordar nuevamente, es necesario para el bienestar de las personas, pero no tiene valor económico y social.

Estos imaginarios son los que subyacen tras la concepción del trabajo en el sector de empleo de atención a las personas, un empleo que se entiende como prolongación de las tareas domésticas y familiares y, por lo tanto, como ocupaciones que tienen «justificada» la baja calidad, las pésimas condiciones laborales y los bajos salarios. Por lo tanto, son empleos y servicios que están pensados como un servicio no cualificado, lógicamente situado en el seno de la precariedad, lo cual limita sus posibilidades de mejora. En realidad, en la casi totalidad de municipios catalanes no se considera que el empleo de calidad sea un elemento imprescindible para proporcionar unos servicios SAD de calidad. O, por lo menos, no se dan los pasos oportunos para favorecer un empleo en los SAD que cumpla estas características, a pesar de que esa mejor cualificación 
y profesionalización del empleo parece un requisito indispensable para la mejora de esos servicios y del bienestar de la población. Hoy por hoy, todo apunta a que estamos en el terreno de lo que «debería ser» y no en la realidad de lo «que es».

Por último, cabe mencionar una circunstancia añadida que, en realidad, interfiere en la situación descrita como un factor no previsto en nuestra investigación. Las políticas municipales de promoción económica/empleo dependen, en gran parte, del modelo de las políticas laborales supramunicipales, un modelo no siempre flexible que impide el desarrollo de políticas propias en los territorios. Así, las políticas de empleo que se llevan a cabo en el territorio están muy vinculadas a las políticas del Servicio de Empleo de Cataluña (SOC), que, a su vez, están muy limitadas por las líneas establecidas por el Ministerio de Trabajo y el Fondo Social Europeo. Son éstas unas entidades que exigen un tipo muy determinado de políticas de empleo, que presionan y constriñen las posibilidades reales de desarrollar políticas laborales innovadoras en los municipios. Es necesario añadir, igualmente, que las vías de definición de los perfiles profesionales escapan también a menudo de las atribuciones del ámbito local. Por lo común, los municipios se limitan a ofrecer los cursos del SOC, que en ningún caso aseguran la inserción laboral posterior. Por otra parte, los municipios, a pesar de detectar las necesidades, no tienen voz en el debate actual sobre las posibilidades de profesionalización del sector. En estos momentos, tanto en Cataluña como en el resto de España, uno de los problemas para la profesionalización del sector es que la formación específica no está plenamente homologada, siendo especialmente notables las carencias en los perfiles profesionales especializados en la gestión y planificación e inspección de los SAD, perfiles que sí se han desarrollado en el campo de la sanidad o de la enseñanza. En los SAD, sólo existen cursos de formación ocupacional, con un requerimiento de horas mínimas que, además de oscilar, presenta fuertes variaciones, y una modalidad de formación profesional que engloba el área sociosanitaria y comunitaria, y no cuenta con el prestigio y atractivo suficiente para interesar al público, joven y femenino, al que va destinado. Así las cosas, cada Ayuntamiento puede marcar en su pliego de condiciones cuál es la titulación mínima requerida para optar a esos empleos y, normalmente, sólo indica el mínimo de horas de formación necesaria que deben tener las trabajadoras contratadas por las empresas que han de prestar el servicio municipal. Por lo tanto, la falta de un marco formativo común y la inconsistencia de la formación existente refuerzan la baja cualificación y el prestigio de esos empleos; en consecuencia, los sitúa muy lejos de la calidad. 


\section{Conclusiones}

El resumen de nuestras conclusiones puede sintetizarse como sigue:

La posible relación que, según nuestras hipótesis de partida, se establece entre el DL y los servicios SAD ha producido un aumento en la creación de empleo, que, sin embargo, no va acompañado de criterios de calidad.

El fomento del empleo en este sector no forma parte de los ejes prioritarios del desarrollo local, porque sólo se contempla como un factor de cohesión social.

Ambas cuestiones impiden que el bienestar cotidiano pueda organizarse socialmente, e inciden en el refuerzo de la estrategia familista a la hora de proveer ese bienestar a la ciudadanía, tanto en Cataluña como en el resto de España.

El detalle de los resultados obtenidos apunta hacia la falta de evidencias claras sobre la existencia de un modelo de desarrollo local alternativo, un modelo que debería centrarse en la promoción del bienestar cotidiano y del empleo de calidad en el sector SAD. Es cierto que se han producido avances importantes, porque los servicios de atención a las personas se han convertido en un área de creciente interés municipal, a diferencia de lo que sucedía hace unas décadas. Pero también es cierto que esta mayor relevancia del sector no tiene una clara relación con el desarrollo local de los municipios, ya que, lejos de afrontar la concepción de este sector como emergente y pionero del DL, es un sector pensado como un nicho de empleo femenino de baja calidad.

El análisis comparativo de entrevistas a personal técnico y político de los tres municipios estudiados ha permitido extraer unas primeras razones explicativas de cuál es y qué ha pasado en el escenario actual de los servicios de cuidado y las políticas locales de bienestar. Una primera constatación es que el SAD está atravesando hoy un momento de tránsito institucional, fruto del despliegue de la Ley de Dependencia española y la Ley de Servicios Sociales catalana, moviéndose en un continuo de asistencialismouniversalización. Esto significa estar a caballo entre la tradición de paliar de forma asistencialista las situaciones de malestar extremo de las personas, y la proactividad, la prevención y la universalización del servicio SAD. Sin embargo, parece detectarse un cierto cambio de mentalidad en torno al modus operandi del Área de servicios sociales y personales y el imaginario social que los ampara, un cambio que abre expectativas respecto a la universalización de los servicios de atención a las personas, pero que todavía no se concreta ni en la forma de financiación ni en términos efectivos de creación de empleo, con un perfil y cualificación convenientemente acreditados.

Se ha comprobado, así mismo, que no se ha generado empleo de calidad en el sector $\mathrm{SAD}$, lo que cuestiona nuestra hipótesis de partida, que vinculaba la creación de empleo de calidad con la promoción de los procesos de desarrollo local. De hecho, con 
el actual sistema se hace difícil establecer un control sobre la inserción laboral obtenida tras los cursos de formación ocupacional promovidos en los municipios.

En este punto, cabe añadir que la carencia de profesionales es una queja recurrente de los responsables y expertos, pero todo indica que la existencia de un perfil profesional de calidad en los SAD queda trabada por una inercia familista y asistencialista, propia de la tradición de regímenes de bienestar mediterráneos, ampliamente aceptada por la sociedad catalana y española. Esta tradición continúa elevando la familia como la principal proveedora y veladora del bienestar cotidiano, una situación calificada de caring culture (Letablier, 2007), en la que los imaginarios colectivos amparan una división sexual del trabajo tradicional donde la «mujer cuidadora» resulta ser la clave del montaje. Tal situación resulta, por el momento, poco permeable para replantear la organización social del cuidado cotidiano de las personas y los cimientos del estado del bienestar.

En relación con la posible existencia de un modelo de desarrollo local, centrado en la promoción del bienestar, no parece que los ayuntamientos analizados hayan apostado por una vía alternativa de DL. Y, pese a reconocer alguna experiencia innovadora, incluso en el propio territorio, no parecen apoyarla porque, probablemente, la mejora del sector SAD no depende únicamente de la política municipal. En este punto, es preciso recordar que, si bien los consistorios pueden desempeñar un papel activo, por tener competencias sobre los SAD, las posibilidades de mejora de dichos servicios están muy vinculadas a la política social y laboral autonómica y estatal.

Finalmente, cabe reseñar una cuestión que resulta clave para dilucidar el escenario aquí presentado: la antonimia implícita en el imaginario sociopolítico entre empleo y bienestar cotidiano, o entre la generación de empleo de calidad y la atención al cuidado de la ciudadanía. La investigación aquí presentada parece mostrar que, de momento, el cuarto pilar del estado del bienestar no se erige como una parcela de creación de empleo de calidad, tal y como vaticinaba el Libro Blanco (Delors, 1994), ya que los SAD no aparecen como una de las prioridades de actuación de las áreas de promoción económica locales. Parece que nos hallamos en una encrucijada de intereses, maneras de pensar y de hacer que, hoy por hoy, presentan una difícil conciliación. Por un lado, el área que promueve los SAD y el bienestar cotidiano en los municipios está poco impregnada por la lógica de creación de empleo, mientras que, simultáneamente, la idiosincrasia de las áreas locales de promoción económica/empleo aún arrastran rasgos del desarrollo local clásico, basado en el desarrollismo y la tradición industrial, lugar donde poca cabida tienen los SAD.

En este sentido, una meta que alcanzar con cierta urgencia en los ayuntamientos es cómo erosionar ese círculo vicioso que aleja a los SAD de una eventual profesionalización e institucionalización. Se trata de una tarea compleja, teniendo en cuenta la fuerte 
carga simbólica de la lógica desarrollista que aún tiñe el desarrollo local en España, así como el imaginario familista y asistencialista que acompaña a los SAD, unos servicios que aún se inscriben simbólicamente en la esfera familiar y bajo la impronta de la domesticidad mediterránea. Podríamos decir que la inclusión del sector del cuidado como pieza clave de las políticas locales de bienestar cotidiano requiere promover un cambio de mentalidades, un cambio que, además de los recursos materiales necesarios, permita vencer o paliar las resistencias culturales derivadas de la tradición familista; un cambio que implica repensar el actual modelo de bienestar, propio de los regímenes de bienestar mediterráneos, y que, por descontado, queda fuera de los límites de la investigación aquí reseñada.

\section{Bibliografía}

Allende, Jose (1995), «Desarrollo sostenible. De lo global a lo local», en Ciudad y territorio: Estudios Territoriales (104), 267-281.

Anderson, Bridget (2000). Doing the dirty work? The global politics of domestic labour. New York: Zed Books.

Balbo, Laura (1994)+ «La doble presencia», en Borderías, Cristina; Carrasco, Cristina y Alemany, Carmen (ed.) Las mujeres y el trabajo: rupturas conceptuales. Barcelona: Icaria-Fuhem.

Bettio, Francesca y Plantenga, Janette (2004). «Comparing care regimes in Europe», en Feminist Economics, 10 (1), 85-113.

Betrio, Francesca; Simonazzi, Annamaria y Villa, Paola (2006). «Change in care regimes and female migration: the 'care drain' in the Mediterranean», en Journal of European Social Policy, 16 (3), 271-285.

Delors, Jacques (1995). Libro Blanco: Crecimiento, competitividad y empleo. Retos y pistas para entrar en el siglo XXI. Comisión de las Comunidades Europeas.

Fraisse, Geneviève (2000). «Servidumbre, servicios de proximidad y democracia», en Maruani, Margaret: Rogerat, Chantal y Torns, Teresa (ed.), Las nuevas fronteras de la desigualdad. Barcelona: Icaria.

Gardiner, Jean (2000). «Rethinking self-sufficiency: employment, families and welfare», en Cambridge Journal of Economics (24), 671-689.

González, José Manuel (2000). «Desarrollo local y medio ambiente», en Pérez, Bartolomé y Carrillo, Emilio (ed.), Desarrollo local. Manual de uso. Madrid: Federación Andaluza de Municipios y Provincias.

Lewis, Jane (2001). «The decline of male breadwinner model: implications for work and care», en Social Politics 8 (2), 152-169. 
Letablier, Marie-Thérèse (2007). «El trabajo de “cuidados” y su conceptualización en Europa», en Prieto, Carlos (ed.) Trabajo, género y tiempo social. Madrid: Hacer.

Ley 39/2006, de 14 de diciembre, de Promoción de la Autonomía Personal y Atención a las personas en situación de dependencia. BOE núm. 299 del viernes 12 de diciembre de 2006.

Llei 12/2007, d'11 d'octubre, de serveis socials. DOGC núm. 4990, 18 de octubre de 2007.

Lope, Andreu; Gibert, Francesc y Recio, Albert (2005). Propostes per l'establiment d'un protocol sobre ocupació en la prestació dels serveis d'Atenció Domiciliaria de Manlleu. Barcelona: QUIT.

Miguélez, Faustino; Lope, Andreu, y Olivares, Isabel (2006). Eldery care sector in Spain. Proyecto Dynamo-QUIT (impreso).

Miguélez, Faustino y Lope, Andreu (2007). «El empleo en la atención a las personas mayores dependientes: necesidades, calidad, profesionalidad y regulación del empleo», Ponencia en el IX Congreso Español de Sociología, Barcelona.

Pateman, Carole (1994). El contrato sexual. Barcelona: Anthropos.

Renner, Michael (2000). «Crear empleos, conservar el medio ambiente. Situación del mundo», en La situación del mundo: informe anual del Worldwatch Institute sobre progreso hacia una sociedad sostenible. Barcelona: Icaria Editorial.

Torns, Teresa (2008), «El trabajo y el cuidado: cuestiones teórico-metodológicas desde la perspectiva de género», en Empiria (15), 53-73.

Ungerson, Clare (2005). «Care, work and feeling», en The Sociological Review, 53 (2), 188-203.

VÁzquez, Antonio (1999). Desarrollo, redes e innovación: lecciones sobre desarrollo endógeno. Madrid: Ediciones Pirámide. 